\title{
Contribution to the knowledge of the Leucospidae, Sapygidae and Vespidae (Apocrita: Hymenoptera) fauna from Bingöl, Diyarbakır and Muş provinces of Turkey
}

\author{
EMÍN KAPLAN ${ }^{1}$, EROL YILDIRIM ${ }^{2,3}$
}

\footnotetext{
${ }^{1}$ Bingöl University, Faculty of Agriculture Department of Plant Protection, 12000 Bingöl, Turkey, email: eminkaplan021@gmail.com, ekaplan@bingol.edu.tr

${ }^{2}$ Atatürk University, Faculty of Agriculture, Department of Plant Protection, 25240, Erzurum, Turkey.

${ }^{3}$ Atatürk University, Biodiversity Aplication and Research Center, Erzurum, Turkey. email: eyildirimerol@hotmail.com
}

\begin{abstract}
Kaplan, E. \& Yildirim, E.: Contribution to the knowledge of the Leucospidae, Sapygidae and Vespidae (Apocrita: Hymenoptera) fauna from Bingöl, Diyarbakır and Muş provinces of Turkey.

Abstract: This study is based on material Leucospidae, Sapygidae and Vespidae specimens collected from Bingöl, Diyarbakır and Muş provinces of Turkey between 2016 and 2020. Two species from one genus of Leucospidae, three species from two genera of Sapygidae and 43 species from 18 genera in four subfamilies of Vespidae are recorded. In total, 48 species belonging to 21 genera are listed. Photographs of the identified species are provided.
\end{abstract}

Keywords: Hymenoptera, Apocrita, Leucospidae, Sapygidae, Vespidae, new records, Bingöl, Diyarbakır, Muş, Turkey,

\section{Introduction}

Numerous neoendemism (LJUBOMIROV \& YILDIRIM 2008) were resulted by variable topography of Turkey with isolated highlands formed along the Turkish border with Georgia, Armenia and Iran emerged after the Pliocene. The Hymenoptera fauna of Turkey contains high diversity of species similarly to other mediterranean countries, which are well known for their high biodiversity (KAPLAN et al. 2018).

The Vespidae is rather big family of Aculeate wasps belonging to the superfamily Vespoidea. The data from previous studies, a total 269 species and 32 subspecies in 53 genera of the subfamilies Vespinae, Polistinae, Eumeninae, and Masarinae belonging to Vespidae are recorded from Turkey (YILDIRIM 2012, YILDIRIM \& GUSENLEITNER 2012, 2015). The families of Leucospidae and Sapygidae are members of the smallest of the Apocrita suborder. A total 15 species in six genera of the subfamilies Fedtschenkiinae and Sapyginae belonging to Sapygidae are recorded from Turkey (YILDIRIM 2016). The Leucospidae family is one of the smallest of the Chalcidoidea families. This family have 143 species described in 4 genera in the world fauna (Noyes 2018, Lima \& Dias 2018). 


\section{Material and methods}

The material was collected at numerous localities in Bingöl, Diyarbakır and Muş provinces in Turkey between 2016 and 2020 (Fig. 1). The samples were collected by insect net. Identification was made by Prof. Dr. Erol Yıldırım. The specimens and, localities are given in alphabetical order in the list below. The collected specimens were deposited in the insect collection of the Department of Plant Protection, Faculty of Agriculture, Bingöl University.

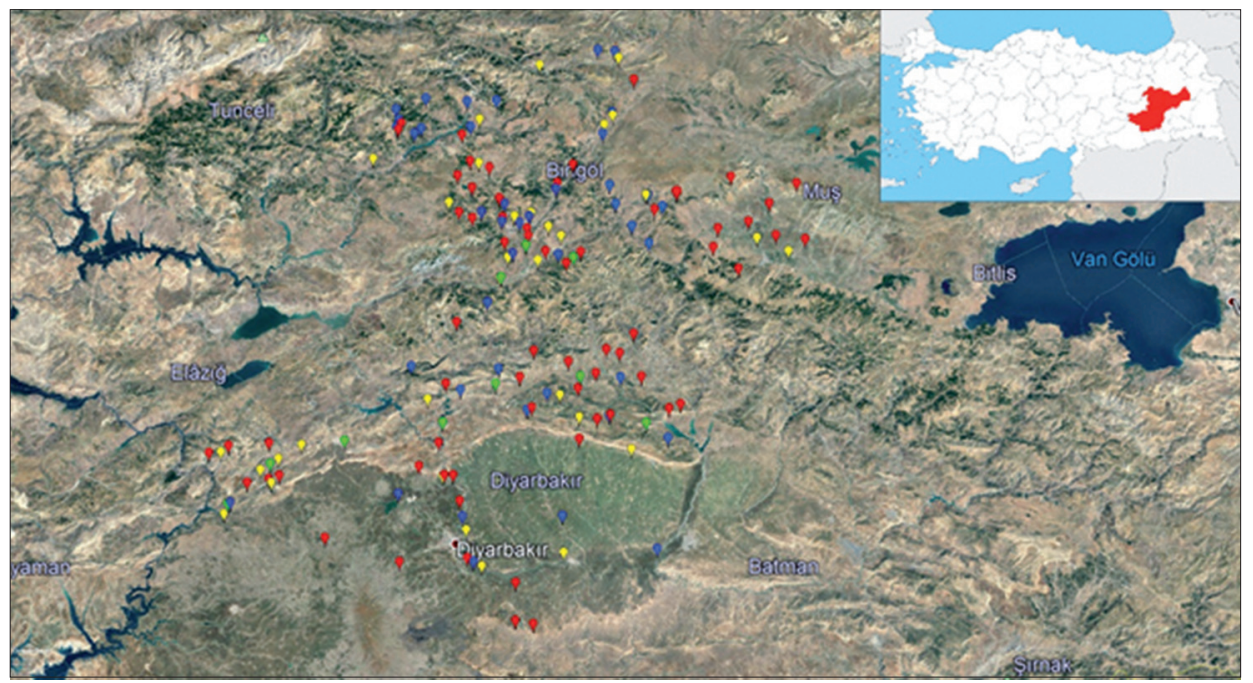

Fig. 1: Map of the investigated area in Turkey (2016, 2017: $0,2018: 0,2019: 0 ; 2020: 0)$

\section{Results}

In this study, two species from one genera of Leucospidae, three species from two genera of Sapygidae and 43 species from 18 genera in four family of Vespidae are listed from Turkey. In total, 48 species belonging to 21 genera of Apocrita are recorded from Turkey.

Family: Leucospidae Fabricius, 1775

Genus: Leucospis Fabricius, 1775

\section{Leucospis dorsigera Fabricius, 1775 (Fig. 2a)}

Material examined: Bingöl: Sudüğünü, N 3903' 29.55", E 40² 24' 39.20", 1654 m, 20.VII.2017, ○; Karlıva, Toklular, N $39^{\circ} 15^{\prime} 57.61^{\prime \prime}$ E E 40 59' 13.07", 1792 m, 03.VI.2018, ôं; Diyarbakır: Lice, Yolçatı, N $38^{\circ} 22^{\prime}$ 39.71", E 40 40' 50.17", 1015 m, 18.IV.2018, ô.

Leucospis intermedia Illiger, 1807 (Fig. 2b)

Material examined: Bingöl: Çavuşlar, N $38^{\circ} 56^{\prime}$ 11.52", E 40 45' 49.57", 1363 m, 26.V.2019, ふ઼; Çayağz1, N

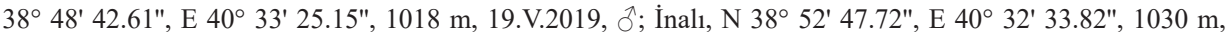
20.V.2019, +; Karlıova, Yeniköy, N 39॰ 11' 40.44", E 40 55' 37.11", 1711 m, 31.V.2019, q; Diyarbakır: Silvan, Dolapdere, N $38^{\circ}$ 18' 30.36", E 40 53' 09.11", 910 m, 05.IV.2019, ふ̋. 
Family: Sapygidae Latreille, 1810

Genus: Polochrum Spinola, 1805

Polochrum repandum Spinola, 1805 (Fig. 2c)

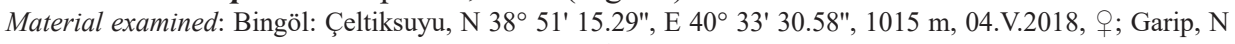

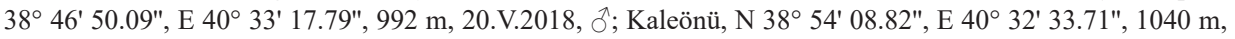
05.V.2018, o;; Küçükterkören, N $38^{\circ}$ 51' 01.40", E 40³0' 02.62", 1182 m, 19.V.2019, q.

\section{Genus: Sapyga Latreille, 1796}

Sapyga caucasica schevyrevi F. Moravitz, 1889 (Fig. 2d)

Material examined: Bingöl: Çiçekyayla, N 38 49' 22.83", E 40² 27' 48.78", 1511 m, 23.V.2019, đ̊’; Gözeler, N $38^{\circ} 51^{\prime} 17.14 "$ ", E 40 40' 36.42", 1153 m, 25.V.2019, ơ’; İnalı, N $38^{\circ} 53^{\prime}$ 27.46", E 40 31' 29.97", 1073 m, 26.V.2019, o; Kırkağ1l, N $38^{\circ}$ 54' 48.08", E 40²2' 42.70", 1731 m, 27.V.2017, ơ’; Sancak, N 39 05' 37.64", E $40^{\circ} 22^{\prime}$ 39.54", 1586 m, 26.V.2018, q; Sarıçiçek, N 38 52' 54.56", E 40 34' 02.26", 1025 m, 04.V.2018, , ;

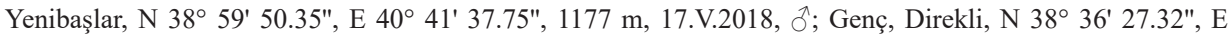

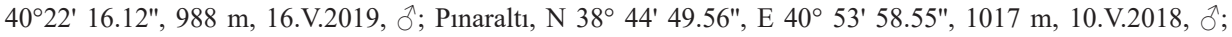
Solhan, Dilektepe, N $38^{\circ}$ 56' 58.39", E 4100' 05.06", 1288 m, 02.VI.2018, q; Diyarbakır: Çermik, Toplu, N $38^{\circ} 06^{\prime}$ 16.37", E $39^{\circ} 33^{\prime}$ 48.02", 779 m, 15.IV.2018, ㅇ; Lice, Dernek, N 38²6' 45.49", E 40 36' 25.47", 938 m, 13.V.2017, ふै; Silvan, Fisat, N $38^{\circ}$ 16' 14.90", E 40 55' 58.22", 999 m, 05.IV.2019, ․

Sapyga singla Kurzenko 1994 (Fig. 2e)

Material examined: Bingöl: Solhan, Dilektepe, N $38^{\circ}$ 57' 17.43", E 40 59' 15.07", 1291 m, 24.V.2018, 우.

Family: Vespidae Latreille, 1802 Subfamily: Eumeninae

Genus: Ancistrocerus Wesmael, 1836

Ancistrocerus auctus Fabricius, 1793 (Fig. 2f)

Material examined: Bingöl: Solhan, Arakonak, N 38 57' 26.93", E 41º 06' 54.36", 1612 m, 02.VI.2018, §̂.

Ancistrocerus claripennis ponticus Giordani Soika, 1970 (Fig. 2g)

Material examined: Bingöl: Aşağıçarşı, N 38 53' 16.22", E 40²9' 38.99", 1130 m, 19.IV.2017, ơં; Gökçeli, N $38^{\circ} 53^{\prime}$ 01.85", E 40 44' 42.29", 1346 m, 25.V.2019, đ̊; Genç, Sülünkaş, N 38 49' 38.68", E 40 56' 57.07",

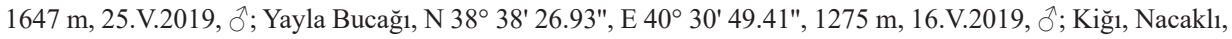

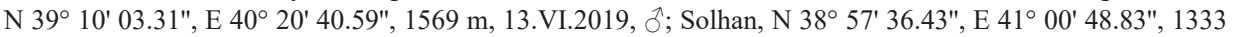

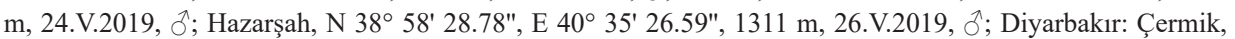
Göktepe, N $38^{\circ}$ 05' 43.12", E 39²2' 31.06", 716 m, 24.III.2019, ô’; Dicle, Baltac1, N 38 23' 17.24", E 380 23' 17.24", 903 m, 27.III.2019, ô’; Bahçedere, N $38^{\circ}$ 18' 52.41", E 40 02' 11.77", 802 m, 28.III.2019, ô’; Hani, Uzunlar, N $38^{\circ}$ 24' 34.34", E 40²7' 03.41", 923 m, 27.III.2019, ô.

Ancistrocerus dusmetiolus (Strand, 1914) (Fig. 2h)

Material examined: Bingöl: Gümüşlü, N 38 45' 66.81", E 40²8' 49.70", 1087 m, 06.V.2018, ふૈં; Solhan, Mutluca, N 3901' 10.17", E 40 52' 01.39", 1631 m, 26.V.2019, ô.

Ancistrocerus parietum (Linnaeus, 1758) (Fig. 2i)

Material examined: Bingöl: Kuşburnu, N $38^{\circ}$ 54' 19.64", E 40 48' 13.49", 1584 m, 25.V.2019, §̂.

Genus: Antepipona Saussure, 1855

Antepipona deflenda (Saunders, 1853) (Fig. 2j)

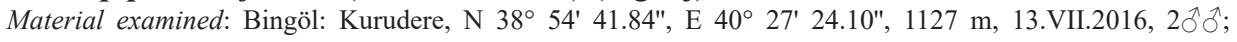


Yoldaşan, N $38^{\circ}$ 43' 88.00", E 40²9' 15.49", 997 m, 05.X.2017, đ̊; Solhan, Yenibaşak Bucağı, N $38^{\circ} 48^{\prime}$

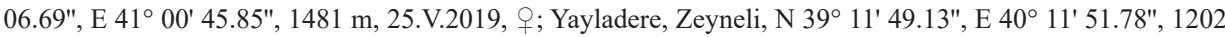
m, 13.VI.2019, ふै.

Antepipona orbitalis orbitalis (Herrich-Schaeff er, 1839) (Fig. 2k)

Material examined: Bingöl: Kardeşler, N 38 54' 48.27", E 40 40' 05.08", 1195 m, 26.V.2019, đ̃.

Genus: Cephalochilus Blüthgen, 1939

Cephalochilus draco Giordani Soika, 1970 (Fig. 21)

Material examined: Bingöl: Çeltiksuyu, N $38^{\circ}$ 52' 13.72", E 40 34' 06.19", 1019 m, 20.VII.2017, O; Çiçekdere, N $38^{\circ} 56^{\prime}$ 57.98", E 40²7'04.84", 1380 m, 20.VII.2017, o ; Çukurca, N $38^{\circ} 57^{\prime} 02.16^{\prime \prime}$, E 40²9' 27.35", 1545 m, 20.VII.2017, o; Genç, Meşedal, N $38^{\circ}$ 46' 45.53", E 40³6' 49.91", 1010 m, 21.VII.2017, ふో; Yiğitbaş1, N $38^{\circ} 44^{\prime} 48.62 "$ " E 40 45' 47.77", 1695 m, 21.VII.2017, ô.

\section{Genus: Eumenes Latreille, 1802}

Eumenes dubius de Saussure, 1852 (Fig. 2m)

Material examined: Bingöl: Karlıova, Toklular, N 39 16' 10.81", E 40 59' 88.91", 1804 m, 12.VII.2016, O’; Diyarbakır: Yukarıkılıçtaşı, N $37^{\circ}$ 57' 18.21", E 40 15' 22.53", 608 m, 21.III.2019, o.

Eumenes mediterraneus Kriechbaumer, 1879 (Fig. 2n)

Material examined: Bingöl: Düzyayla, N 38 48' 05.38", E 40²8' 57.28", 1373 m, 06.V.2018, ô’; Kurudere,

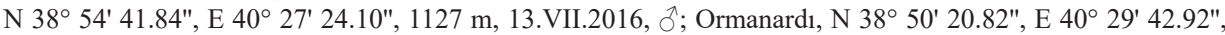

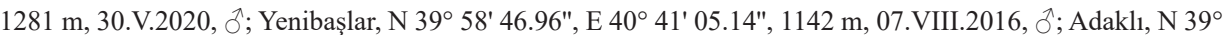

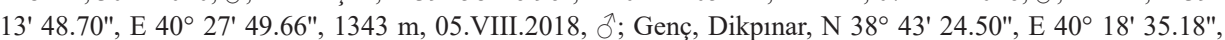

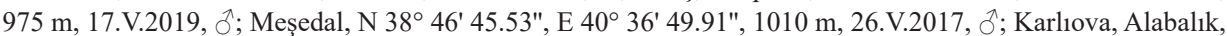
N 39॰ 18' 18.40", E 41 09' 28.08", 1785 m, 04.VI.2018, ô; Ortaköy, N 39²4' 01.75", E 40 53' 40.88", 1929

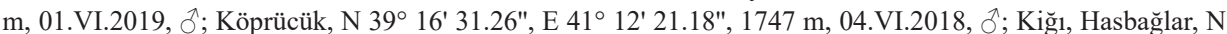
$39^{\circ} 10^{\prime} 52.05^{\prime \prime}$, E $40^{\circ} 22^{\prime} 39.10^{\prime \prime}, 1605$ m, 05.VIII.2016, ő; Solhan, Arakonak, N $38^{\circ} 58^{\prime} 40.46^{\prime \prime}$, E $41^{\circ} 08^{\prime}$

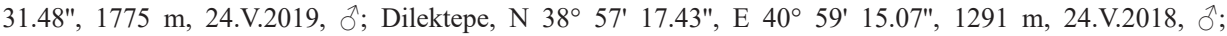
Şimşirpınar, N $38^{\circ}$ 56' 26.96", E 41 04' 38.92", 1555 m, 02.VI.2018, đ̊’; Diyarbakır: Dicle, N 37 53' 24.16",

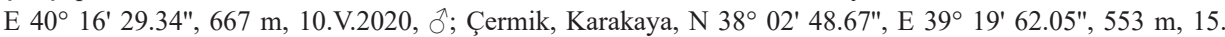

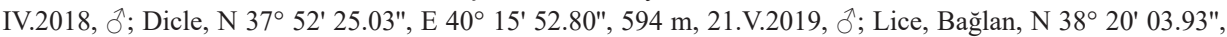

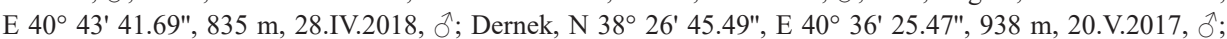
Muş: Mercimekkale, N $38^{\circ} 54^{\prime}$ 43.90", E 41³0' 51.07", 1152 m, 06.VI.2016, ô’; Varto, Bağiçi, N 390 05' 41.68", E 41³1' 31.93", 1332 m, 06.VIII.2016, ふึ.

Eumenes pedunculatus (Panzer, 1799) (Fig. 2o)

Material examined: Bingöl: Akdurmuş, N 38 50' 40.56", E 40² 28' 30.44", 1467 m, 23.V.2019, đ̊; Çeltiksuyu, N

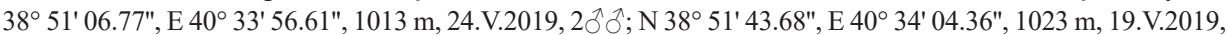

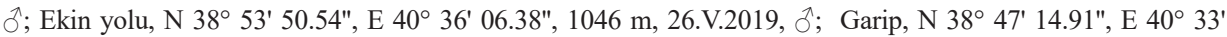

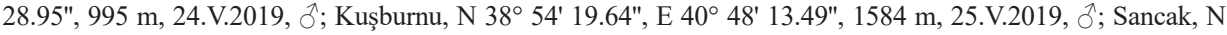

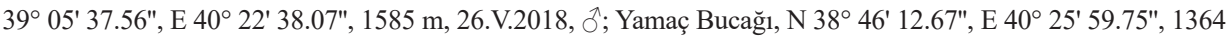

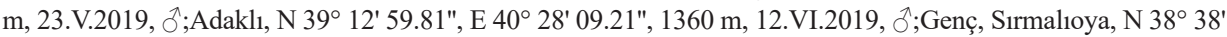

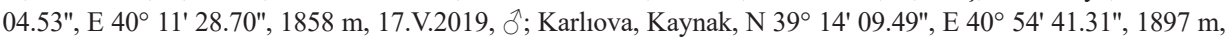

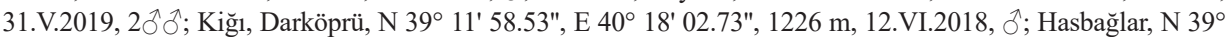
10' 52.05", E 40²2' 39.10", 1605 m, 05.VIII.2016, đ̊; Solhan, Çermük, N 38 59' 39.49", E 40 50' 40.75", 1776

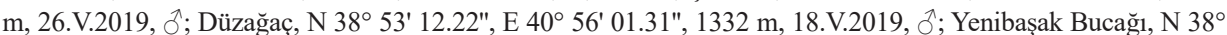
48' 06.69", E 4100' 45.85", 1481 m, 25.V.2019, ỏ; Yayladere, Güneşlik, N 39 12' 01.30", E 40 10' $43.52^{\prime \prime}, 1371$

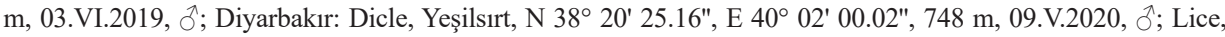

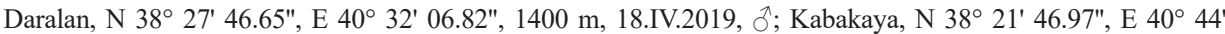

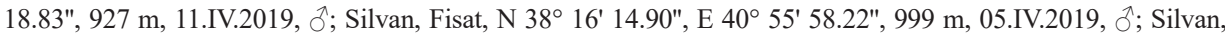
Boyunlu, N $38^{\circ}$ 14' 17.19", E 40 58' 48.17", 991 m, 22.V.2020, ふૈ. 

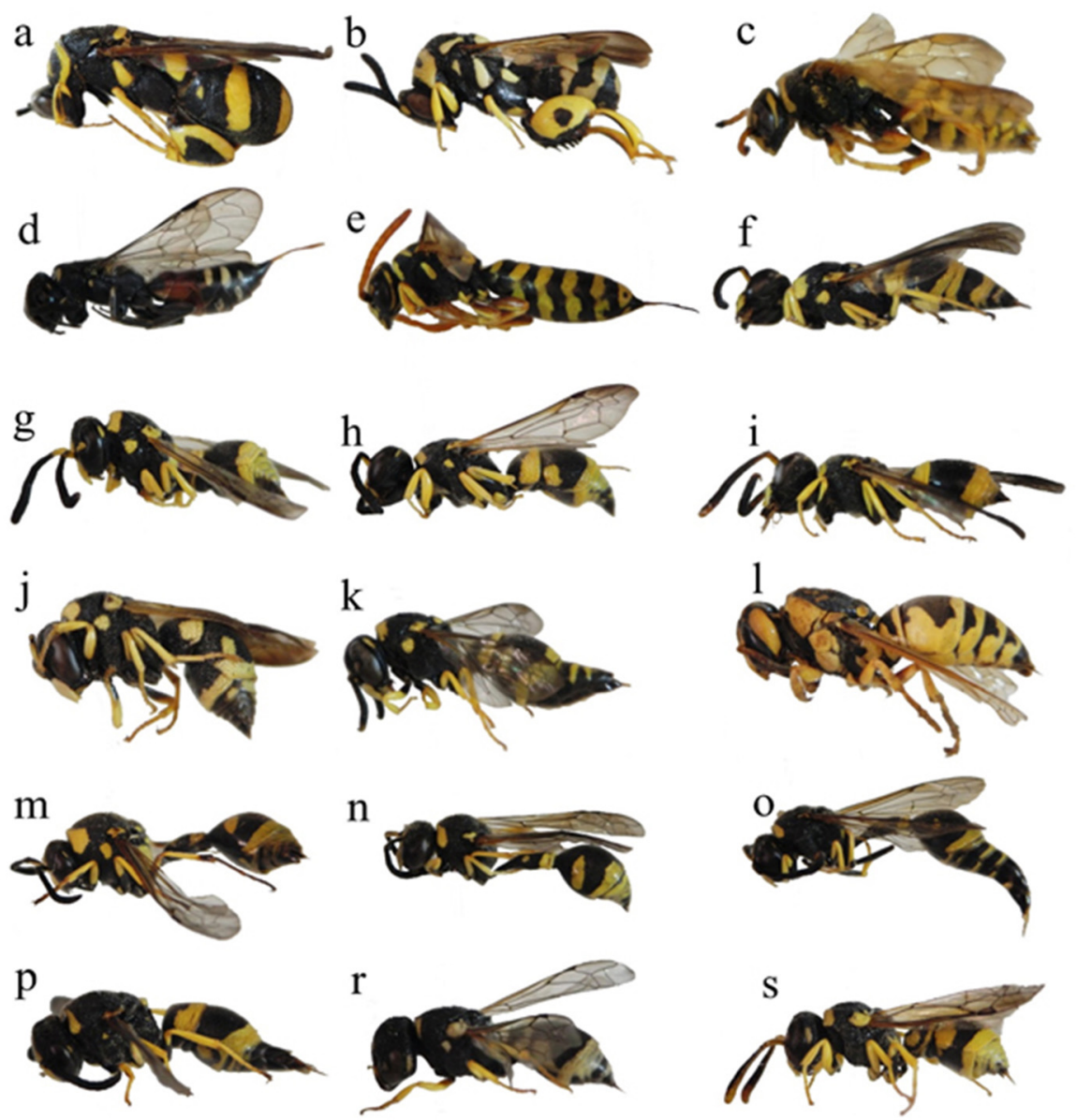

Fig. 2: a) Leucospis dorsigera ( $९)$; b) L. intermedia ( $९)$; c) Polochrum repandum ( $९)$; d) Sapyga caucasica schevyrevi ( + ); e) S. singla ( + ); f) Ancistrocerus auctus ( $\bigcirc^{\text {) }}$; g) A. claripennis ponticus (ठ); h) A. dusmetiolus (đ); i) A. parietum (đ); j) Antepipona deflenda (đ); k) A. orbitalis orbitalis ( $\left({ }^{-}\right)$; l) Cephalochilus draco $(+)$; m) Eumenes dubius $(+)$; n) E. mediterraneus

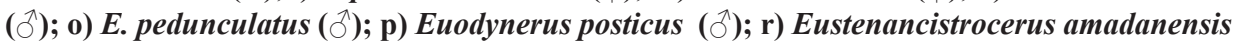
( $\left(\right.$ ); s) Eustenancistrocerus jerichoensis iconius $\left({ }^{\Uparrow}\right)$ 
Genus: Euodynerus Dalla Torre, 1904

Euodynerus posticus (Herrich-Schäffer, 1841) (Fig. 2p)

Material examined: Bingöl: Çayağzı, N $38^{\circ} 48^{\prime} 12.13^{\prime \prime}$, E 40³ 33' 21.90", 1003 m, 20.V.2018, ô; Karlıova,

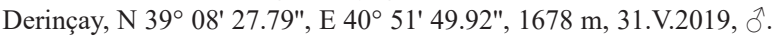

\section{Genus: Eustenancistrocerus Blüthgen, 1938}

Eustenancistrocerus (Parastenancistrocerus) amadanensis (Saussure, 1856) (Fig. 2r) Material examined: Bingöl: Çeltiksuyu, N 38 52' 57.59", E 40 35' 21.66", 1045 m, 20.V.2017, o+; Genç,

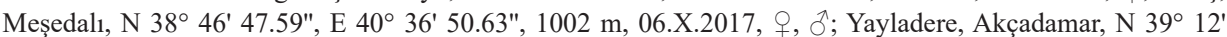
11.02", E 40 04' 08.93", 1385 m, 13.VI.2019, 3̊; Diyarbakır: Köprübaşı, N $38^{\circ} 00^{\prime} 54.22^{\prime \prime}$, E 40² 21' 58.13", 608 m, 21.V.2020, ㅇ; Dicle, Bultac1, N 38² 23' 17.24", E 38² 23' 17.24", 903 m, 27.III.2019, 우.

Eustenancistrocerus (Eustenancistrocerus) jerichoensis iconius Blüthgen, 1957 (Fig. 2s) Material examined: Bingöl: Kurudere, N $38^{\circ}$ 54' 41.84", E 40 27' 24.10", 1127 m, 13.VII.2016, ơ; Yukarıpınar,

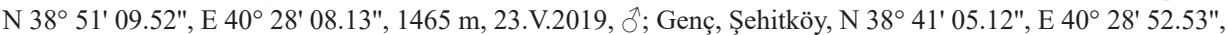
1198 m, 16.V.2019, ô; Yayladere, Doyucak, N 390 09' 46.49", E 40 64' 27.75", 1462 m, 13.V.2019, ô.

\section{Genus: Gymnomerus Blüthgen, 1938}

\section{Gymnomerus leavipes (Shuckard, 1837) (Fig. 3a)}

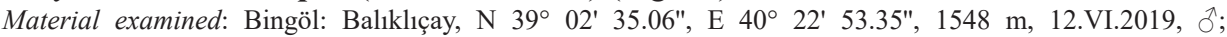

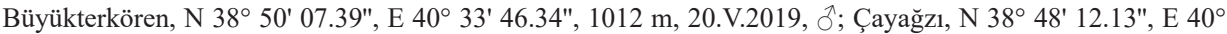

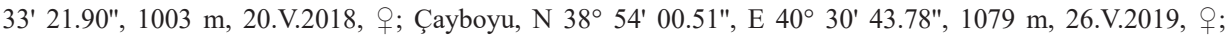

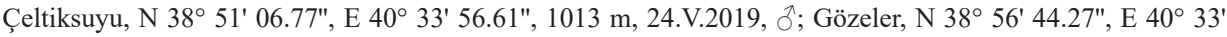

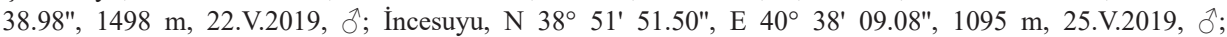

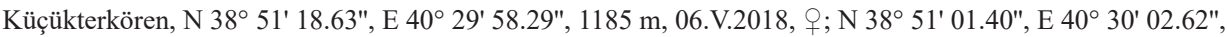

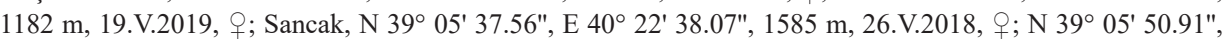

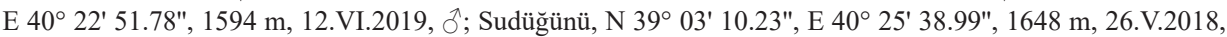

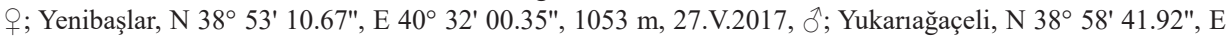

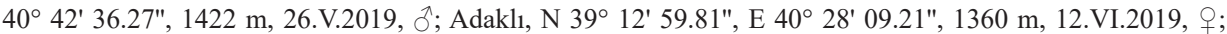

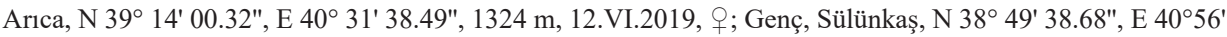

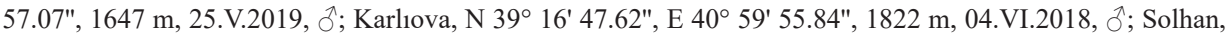

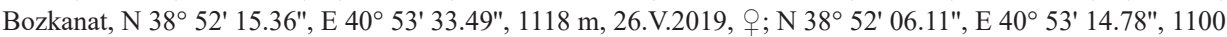

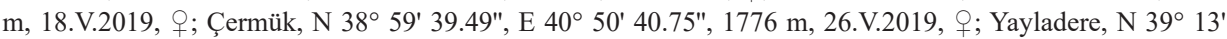

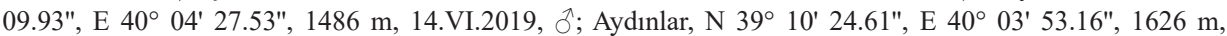

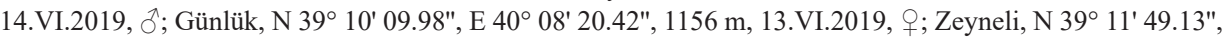

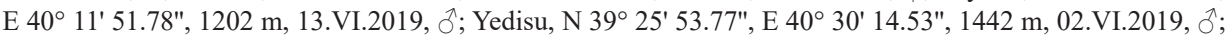

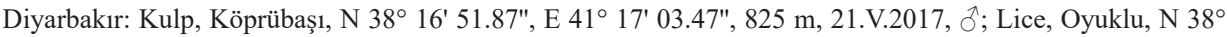
$12^{\prime} 32.95^{\prime \prime}$, E $39^{\circ} 22^{\prime} 09.37^{\prime \prime}, 1007$ m, 24.III.2019, ô.

\section{Genus: Jucancistrocerus Blüthgen, 1938}

Jucancistrocerus jucundus (Mocsáry, 1883) (Fig. 3b)

Material examined: Bingöl: Çeltiksuyu, N 38 51' 44.46", E 40³4' 06.40", 1017 m, 20.V.2018, ô; İnalı, N

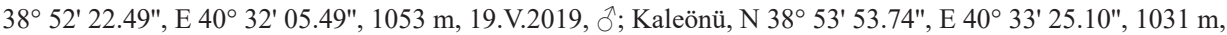

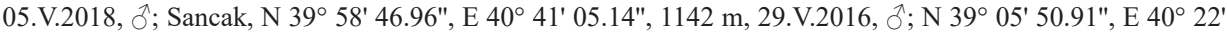
51.78", 1594 m, 12.VI.2019, o ; Sarıçiçek, N 38 53' 16.55", E 40 53' 16.55", 1028 m, 04.V.2018, ô; Adaklı,

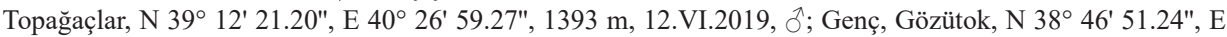

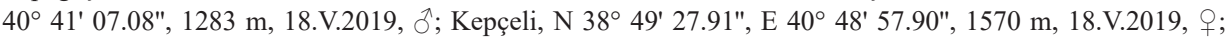
Pinaraltı, N $38^{\circ} 44^{\prime} 49.56^{\prime \prime}$, E $40^{\circ} 53^{\prime} 58.55^{\prime \prime}, 10321$ m, 10.V.2018, ớ; Sülünkaş, N $38^{\circ} 49^{\prime} 38.68^{\prime \prime}$, E $40^{\circ} 56^{\prime}$ 57.07", 1647 m, 25.V.2019, ơ; Karlova, Dörtyol, N 39² 22' 33.58", E 40 58' 16.63", 1813 m, 06.VI.2018, 亦; 

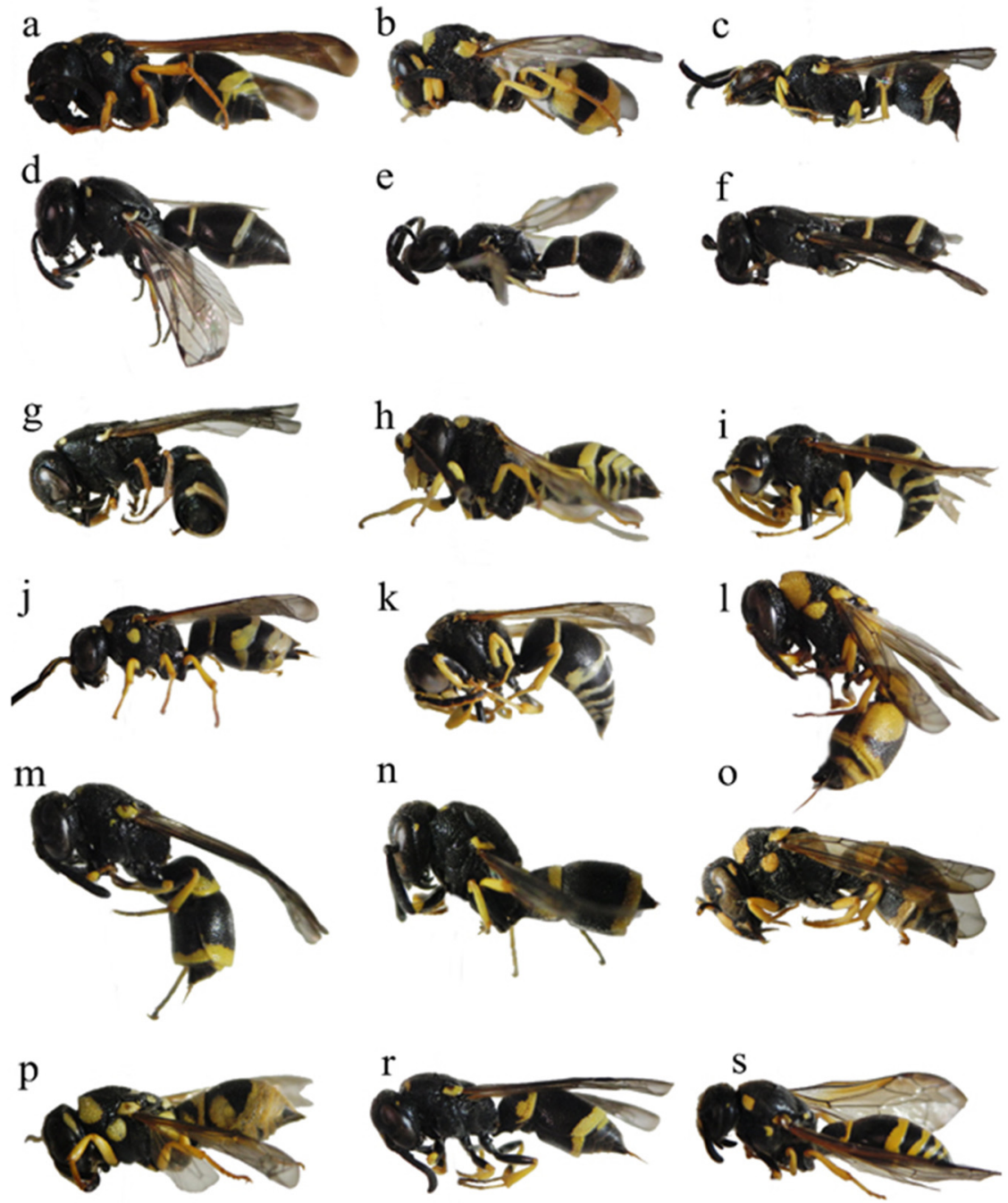

Fig. 3: a) Gymnomerus leavipes ( + ); b) Jucancistrocerus jucundus ( $९)$; c) Leptochilus regulus

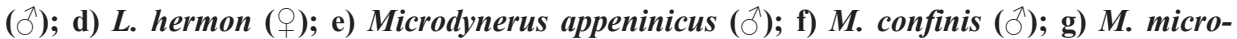
dynerus (); h) Odynerus albopictus $\left({ }^{\lambda}\right)$; i) $O$. femoratus $\left({ }^{\lambda}\right)$; j) 0 . melanocephalus armeniacus (†); k) O. poecilus (つ)); l) Stenodynerus aequisculptus (+); m) S. bluethgeni (†); n) $S$. chevrieranus (†); o) S. simulatus (ð); p) Syneuodynerus egregius $(+)$; r) Symmorphus bifasciatus ( + ); s) S. crassicornis ( + ) 


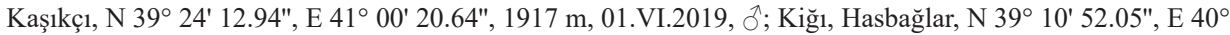

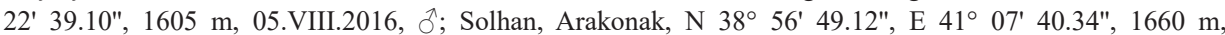

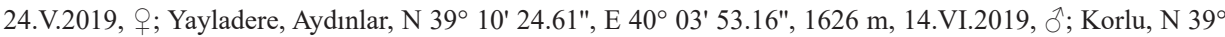
10' 36.36", E 40 09' 19.47", 1231 m, 13.VI.2018, ô’; Diyarbakır: Çüngüş, Yenice, N 38 17' 05.87", E 39 $15^{\prime}$ 28.49", 1209 m, 20.IV.2018, ふ̊; Ergani, Ahmetli Bucağ1, N 38 11' 30.97", E 39 50' 35.49", 798 m, 25.IV.2020, O; Lice, Çavundur, N $38^{\circ}$ 19' 34.16", E 40 40' 31.58", 975 m, 01.IV.2019, đ̊; Muş: Karabey, N 38 55' 26.60", E $41^{\circ} 08^{\prime} 34.76^{\prime \prime}, 1771 \mathrm{~m}, 25 . \mathrm{V} .2018$, ふิ?.

\section{Genus: Leptochilus Saussure, 1853}

\section{Leptochilus (Lionotulus) hermon Gusenleitner, 1971 (Fig. 3d)}

Material examined: Bingöl: Sarıçiçek, N $38^{\circ} 52^{\prime}$ 54.56", E 40 34' 02.26", 1025 m, 04.V.2018, +; Adaklı, Karaçubuk, N $39^{\circ}$ 11' 48.76", E 40² 28' 28.42", 1418 m, 06.VII.2018, ふ̊’; Genç, Dikköy, N 38 48' 51.40", E $40^{\circ} 40^{\prime}$ 19.68", 1019 m, 29.V.2020, ð’; Karlıova, Yeniköy, N 39 11' 50.05", E 40 56' 07.35", 1748 m, 03.

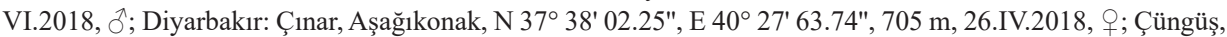
Malkaya, N $38^{\circ}$ 14' 19.47", E 39 16' 37.75", 1136 m, 20.IV.2018, q; Ergani, Yolköprü, N 38 13' 28.02", E $39^{\circ} 38^{\prime} 32.90^{\prime \prime}, 905 \mathrm{~m}, 25 . \mathrm{IV} .2020$, ㅇ․

Leptochilus (Neoleptochilus) regulus (Saussure, 1855) (Fig. 3c)

Material examined: Bingöl: Karlıva, Dörtyol, N 39² 22' 33.58", E 40 58' 16.63", 18133 m, 066.VI.2018, §̂.

Genus: Microdynerus Thomson, 1874

Microdynerus appeninicus (Giordani-Soika, 1960) (Fig. 3e)

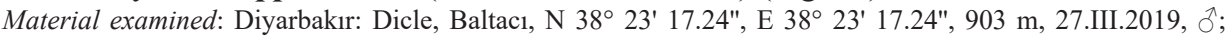
Çüngüş, Oyuklu, N 38 12' 32.95", E 39² 22' 09.37", 1007 m, 24.III.2019, ô.

Microdynerus confinis Gusenleitner, 1979 (Fig. 3f)

Material examined: Diyarbakır: Hazro, Bahçe, N 38 15' 26.47", E 40 47' 33.19", 1088 m, 13.V.2017, ô.

Microdynerus (Alastorynerus) microdynerus (Dalla Torre, 1889) (Fig. 3g)

Material examined: Diyarbakır: Ergani, Çayırdere, N $38^{\circ}$ 11' 50.80", E 39 34' 19.42", 803 m, 21.IV.2017, q.

Genus: Odynerus Latreille, 1802

Odynerus (Odynerus) albopictus Saussure, 1856 (Fig. 3h)

Material examined: Bingöl: Erentepe, N 38 40' 33.74", E 40²8' 26.94", 1462 m, 23.V.2019, ふ̊’; Yayladere,

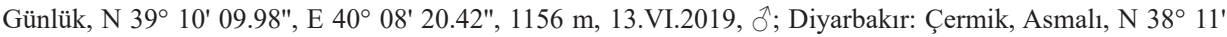
14.72", E 39 31' 34.40", 707 m, 27.IV.2017, ô’; Çüngüş, Akbaşak, N 38 15' 58.97", E 39 18' 26.90", 1385 m, 28.IV.2017, ô; Hazro, Bağyurdu, N 38 14' 25.36", E 40 49' 34.44", 988 m, 15.V.2017, ô.

Odynerus (Odynerus) femoratus de Saussure, 1856 (Fig. 3i)

Material examined: Diyarbakır: Ergani, Bozyer, N $38^{\circ}$ 11' 04.25", E 39 45' 19.47", 798 m, 12.V.2017, ô.

Odynerus (Odynerus) melanocephalus armeniacus (Morawitz, 1885) (Fig. 3j)

Material examined: Bingöl: Balıklıçay, N 39 02' 35.06", E 40 22' 53.35", 1548 m, 12.VI.2019, o;

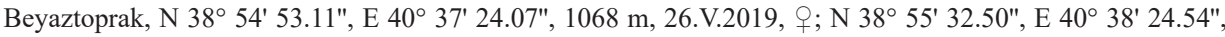
1089 m, 21.V.2019, q; Büyükterkören, N 38 50' 35.45", E 40 34' 13.20", 1017 m, 20.V.2018, . ; N $38^{\circ} 50^{\prime}$

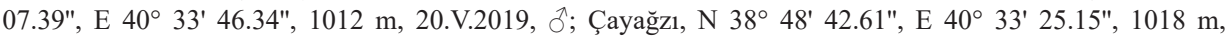

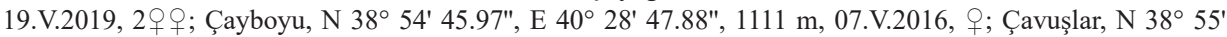

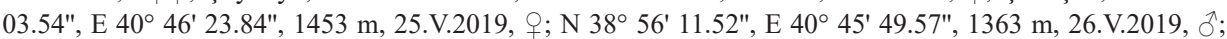
Çeltiksuyu, N $38^{\circ}$ 51' 53.01", E 40 53' 44.99", 1016 m, 29.V.2019, ơ’; Çevrimpınar, N $38^{\circ} 55^{\prime} 49.40^{\prime \prime}$, E 40 22' 12.63", 1318 m, 15.V.2019, +; Çiçekdere, N 38 57' 05.81", E 40²7' 49.41", 1640 m, 22.V.2019, 羊; 


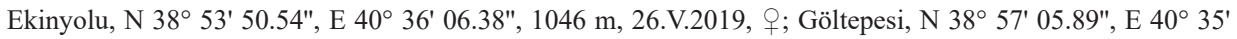
40.86", 1496 m, 22.V.2019, ふ̄; Gümüşlü, N 38 45' 66.81", E 40 28' 49.70", 1087 m, 06.V.2019, , q; Haziran,

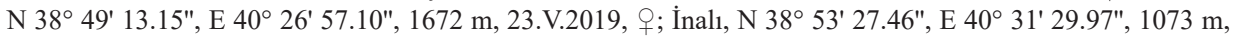

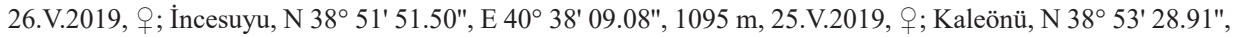

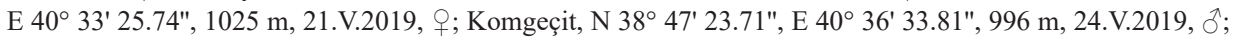

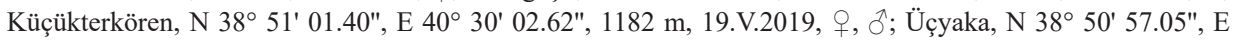
40²7' 09.72", 1701 m, 23.V.2019, O+; Sancak, N 39॰ 58' 46.96", E 40 41' 05.14", 1142 m, 29.V.2016, ô;

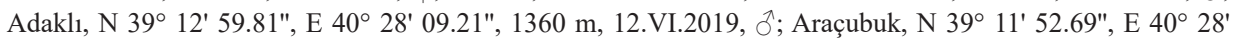

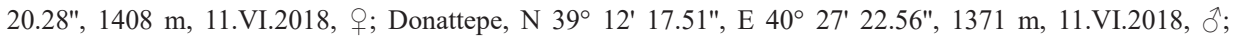
Hasbağlar, N $39^{\circ}$ 11' 01.77", E 40²2' 23.77", 1569 m, 12.VI.2019, o; Genç, Döşekkaya, N $38^{\circ} 38^{\prime} 11.11^{\prime \prime}$, E

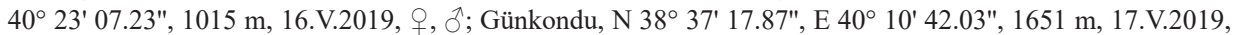

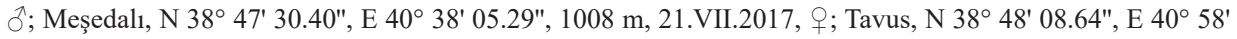
08.43", 1893 m, 25.V.2019, q; Karlıva, N 39 11' 40.44", E 40 55' 37.11", 1711 m, 31.V.2019, q; Bahçeköy, N 39 13' 22.87", E 40 54' 12.20", 1862 m, 01.VI.2019, ơ’; Çilligöl, N 39 10' 59.87", E 40 51' 18.85", 1843

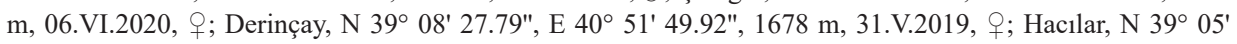
01.63", E 40 49' 21.67", 1417 m, 31.V.2019, 2우, ở; Kaynak, N 39 14' 09.49", E 40 54' $41.31^{\prime \prime}, 1897$ m,

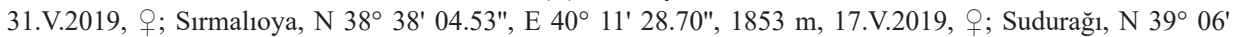
52.44", E 40 51' 06.87", 1648 m, 31.V.2019, ㅇ; Kiğ1, Dallıca, N 39 15' 32.11", E 40 19' 04.67", 1358 m, 13.VI.2019, o; Demirkanat, N 39॰ 12' 25.30", E 40 19' 05.79", 1324 m, 29.V.2017, o, ỏ’; Sabırtaş1, N 39 15' 56.52", E 40 11' 42.22", 1366 m, 13.VI.2019, ㅇ; Solhan, Arakonak, N 38 56' 49.12", E 41 07' 40.34", 1660

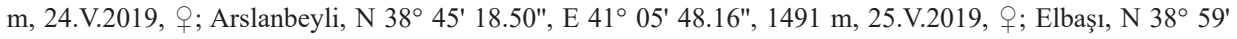

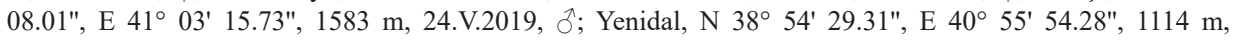

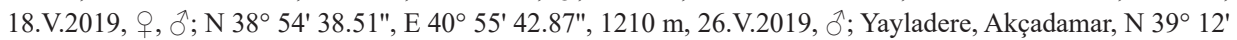
11.02", E 40 04' 08.93", 13385 m, 13.VI.2019, of; Zeyneli, N 39॰ 11' 49.13", E 40 11' 51.78", $1202 \mathrm{~m}$,

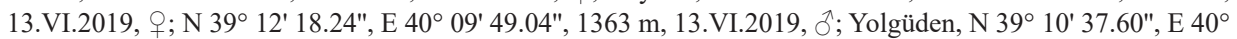
04' 16.59", 1462 m, 13.VI.2019, ô'; Diyarbakır: Avcisuyu, N 38 03' 44.55", E 39 59' 22.13", 741 m, 23. III.2019, o; Köprübaşı, N $37^{\circ}$ 59' 64.39", E 40²2' 59.69", 602 m, 21.III.2019, o,; Oyalı, N 38 07' 59.04", E 40 01' 49.14", 773 m, 30.IV.2017, ð̊; Bismil, Güroluk, N 38 01' 38.44", E 40 35' 18.97", 647 m, 19.III.2019,

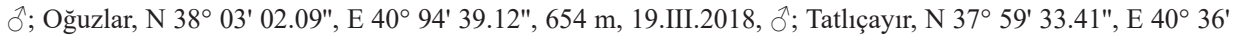
24.42", 620 m, 19.III.2019, ơ’; Çermik, N $38^{\circ}$ 67' 30.98", E 39²7' 42.06", 766 m, 24.III.2019, q; Bademli, N $38^{\circ} 05^{\prime}$ 00.48", E $39^{\circ} 23^{\prime}$ 46.69", 609 m, 27.IV.2017, ㅇ; Bayrak, N 38 03' 42.19", E 39²2' $45.42^{\prime \prime}, 572$ m, 27.IV.2017, o; Gönülaçan, N $38^{\circ}$ 01' 44.96", E 39²0' 49.48", 657 m, 27.IV.2017, 2o o ; Gürüz, N 37 59' 49.31", E 39 22' 59.44", 847 m, 27.IV.2017, ơ’; Kayagediği, N $38^{\circ} 09^{\prime}$ 16.86", E 39 34' 28.52", 1085 m, 15.IV.2018, đ̊; Çınar, N $37^{\circ} 38^{\prime}$ 02.25", E 40²7' 53.74", 705 m, 26.IV.2017, ơ; Bozçalı, N 37 45' 19.49", E 40²1' 08.98", 669 m, 30.III.2018, ô'; Çüngüş, Balçılar, N 38 14' 21.81", E 39 27' 05.14", 1036 m, 28.

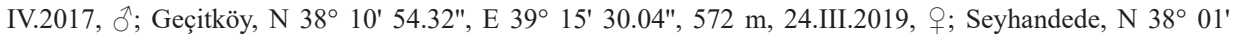

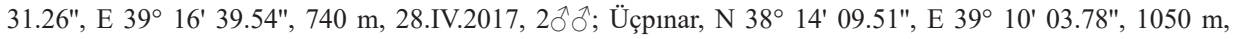

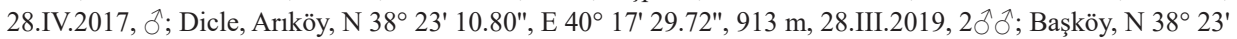

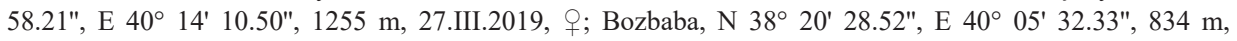

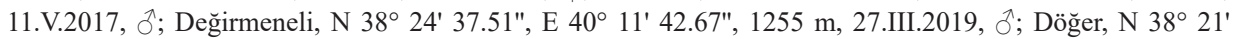
57.31", E 39 13' 41.14", 756 m, 12.V.2017, ơ'; Yeşilsırt, N 38 20' 25.58", E 40 03' 30.70", 797 m, 12.V.2017,

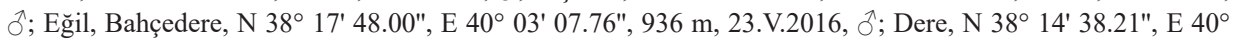

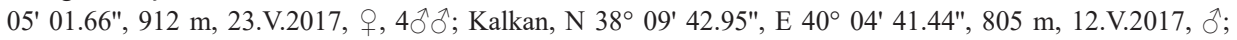
Kazanl1, N $38^{\circ}$ 14' 01.91", E 4009' 13.48", 860 m, 23.V.2016, , ; Konak, N 38 10' 44.39", E 4005' 08.55", 853 m, 12.V.2017, o; Oyalı, N $38^{\circ} 08^{\prime}$ 27.84", E 40 02' 25.72", 874 m, 23.V.2016, o.; N $38^{\circ} 08^{\prime} 27.84^{\prime \prime}$, E $40^{\circ}$

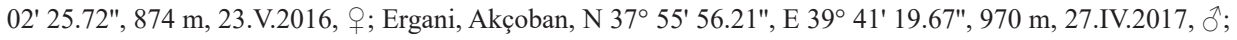

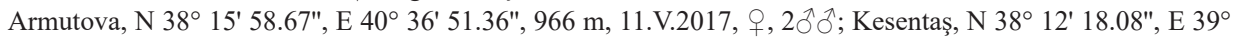
35' 48.49", 875 m, 21.IV.2018, ơ’; Sallar, N 38 16' 01.70", E 39 38' 59.32", 962 m, 11.V.2017, , ; Selmanköy, N $38^{\circ} 08^{\prime}$ 21.64", E 39॰ 41' 11.12", 822 m, 23.V.2016, o ; N 38 11' 45.04", E 40 08' 46.13", 809 m, 12.V.2017,

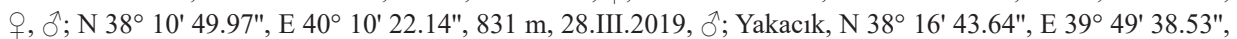

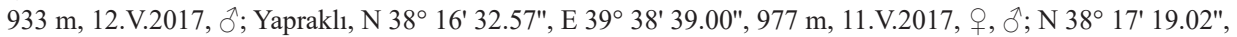

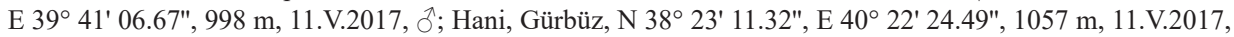

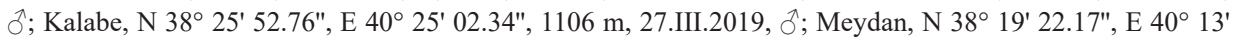
59.83", 761 m, 21.IV.2018, ô’; Süslü, N $38^{\circ} 23^{\prime}$ 27.97", E 40 19' 13.30", 1072 m, 12.V.2017, 2qo ; Hazro, Bağyurdu, N 38 14' 25.36", E 40 49' 34.44", 988 m, 13.V.2017, q; Bahçe, N 38 15' 26.47", E 40 47' 33.19", 
1088 m, 13.V.2017, ô’; Dadaş, N $38^{\circ}$ 16' 01.30", E 40 42' 28.51", 1082 m, 04.IV.2019, đ̊’; Mutluca, N $38^{\circ} 16^{\prime}$ 44.84", E 40 53' 50.71", 1017 m, 13.V.2017, o; Ormankaya, N 38 18' 13.20", E 40 44' 29.38", 1099 m,

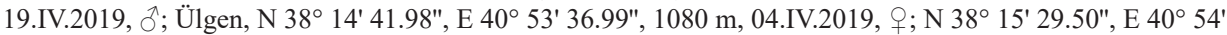
23.83", 1196 m, 26.IV.2019, ở; Kocaköy, Arkbaş1, N 38 16' 17.21", E 40 33' 52.22", 834 m, 28.V.2016, + +; Saklat, N $38^{\circ}$ 19' 44.89", E 40²2' 36.83", 893 m, 01.IV.2019, o;; Kulp, Argunköy, N $38^{\circ} 28^{\prime} 48.51^{\prime \prime}$, E $41^{\circ} 05^{\prime}$

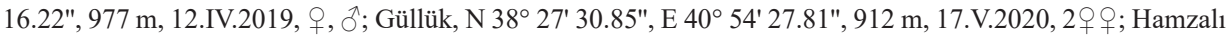

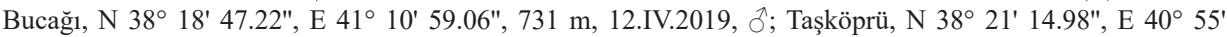
57.30", 860 m, 12.IV.2019, + ; Lice, Abal1, N 38 31' 40.75", E 40 32' 48.20", 962 m, 28.V.2016, o; Acar, N

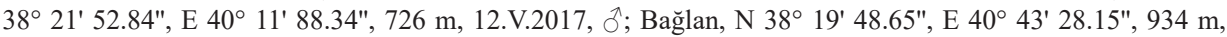

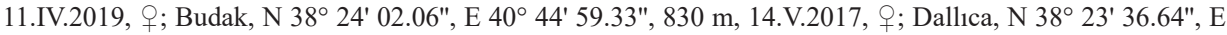
$40^{\circ} 47^{\prime}$ 27.94", 790 m, 20.IV.2019, o.; Gürbeyli, N $38^{\circ}$ 27' 21.78", E 40 42' 52.01", 876 m, 28.V.2016, o ;

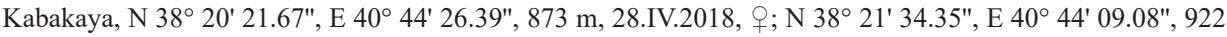

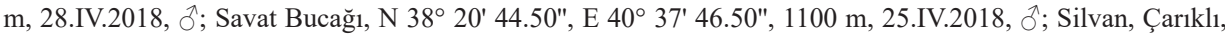
N $38^{\circ} 03^{\prime} 31.08^{\prime \prime}$, E $41^{\circ} 11^{\prime} 32.52^{\prime \prime}, 591$ m, 29.IV.2018, 우; Dolapdere, N 38 18' 30.36", E 40 53'09.11", 910

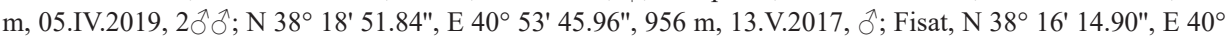

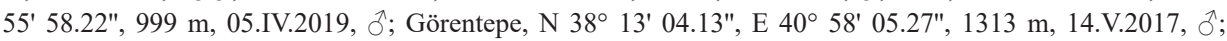

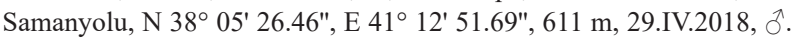

Odynerus poecilus de Saussure, 1856 (Fig. 3k)

Material examined: Diyarbakır: Ergani, Selmanköy, N $38^{\circ} 08^{\prime}$ 21.64", E 39॰ 41' 11.12", 822 m, 23.V.2016, ふ̊’; Kulp, Özbek, N $38^{\circ} 29^{\prime} 13.80^{\prime \prime}$, E 4057' 52.88", 942 m, 21.V.2017, ô.

\section{Genus: Stenodynerus Saussure, 1863}

Stenodynerus aequisculptus (Kostylev, 1940) (Fig. 31)

Material examined: Bingöl: Genç, Gönülaçan, N 38³7' 05.72", E 40 16' 33.17", 1373 m, 21.VI.2017, +.

Stenodynerus bluethgeni van der Vecht, 1971 Fig. 3m)

Material examined: Bingöl: Adaklı, Arıca, N 39 14' 00.32", E 40³1' 38.49", 1324 m, 12.VI.2017, o; Merkez, Ekinyolu, N $38^{\circ}$ 54' 30.05", E 40 35' 01.69", 1044 m, 19.V.2017, q; Yayladere, Merkez, N 39 13' 11.16", E $40^{\circ} 04^{\prime} 10.77^{\prime \prime}, 1553 \mathrm{~m}, 30 . \mathrm{V} .2017$, ․

Stenodynerus chevrieranus (Saussure, 1855) (Fig. 3n)

Material examined: Bingöl: Kartal, N 39 01' 05.56", E 40²3' 28.63", 1675 m, 26.V.2017, ふో; Nacaklı, N 39॰10' 05.38", E 40 21' 33.32", 1537 m, 26.V.2018, ơ; Genç, Meşedalı, N 38 46' 47.59", E 40 36' 50.63",

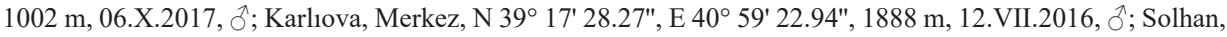

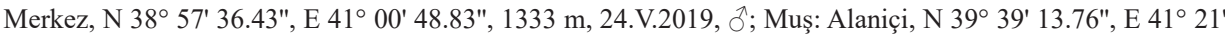
$18.45^{\prime \prime}, 1726 \mathrm{~m}, 25 . \mathrm{V} .2018$, ふे.

Stenodynerus simulatus Gusenleitner, 1981 (Fig. 3o)

Material examined: Bingöl: Kiğı, Hasbağlar, N 39 10' 52.05", E 40²2' 39.10", 1605 m, 05.VIII.2016, ठో.

\section{Genus: Syneuodynerus Blüthgen, 1938}

Syneuodynerus egregius (Herrich-Schäffer, 1839) (Fig. 3p)

Material examined: Bingöl: Büyükterkören, N $38^{\circ}$ 49' 49.46", E 40 34' 28.59", 1009 m, 16.V.2019, , ; İnal1, N $38^{\circ} 52^{\prime} 47.72^{\prime \prime}$, E $40^{\circ} 32^{\prime} 33.82^{\prime \prime}, 1030$ m, 20.V.2019, ô.

Genus: Symmorphus Wesmael, 1836

Symmorphus bifasciatus (Linnaeus, 1761) (Fig. 3r)

Material examined: Bingöl: Çeltiksuyu, N 38 51' 43.68", E 40³4' 04.36", 1023 m, 19.V.2019, o. 
Symmorphus crassicornis (Panzer, 1798) (Fig. 3s)

Material examined: Bingöl: Ekin yolu, N 38 54' 15.91", E 40³4' 33.20", 1044 m, 19.V.2018, o ; Çukurca, N $38^{\circ} 55^{\prime} 13.31^{\prime \prime}$, E $40^{\circ} 31^{\prime} 17.62^{\prime \prime}, 1215$ m, 05.V.2018, o+; Derinçay, N 390 08' 27.79", E 40 51' 49.92", 1678 $\mathrm{m}, 31 . \mathrm{V} .2019$, ㅇ.

Symmorphus gracilis (Brullé, 1832) (Fig. 4a)

Material examined: Bingöl: Genç, Harmancık, N 38 33' 40.32", E 40 16' 39.89", 883 m, 17.V.2019, ố;

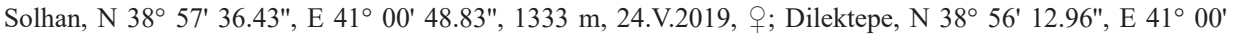
08.44", 1299 m, 24.V.2019, §̂.

\section{Symmorphus murarius (Linnaeus, 1758) (Fig. 4b)}

Material examined: Bingöl: Çevrimpınar, N 38 55' 49.40", E 40²2' 12.63", 1318 m, 15.V.2019, ô'; Adaklı,

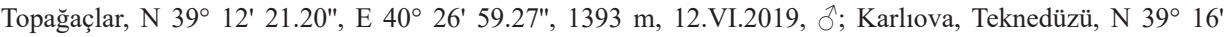
09.65", E 41 13' 20.05", 1691 m, 04.VI.2018, ô; Kiğı̆, Dallıca, N 39º 15' 32.11", E 40 19' 04.67", 1358 m,

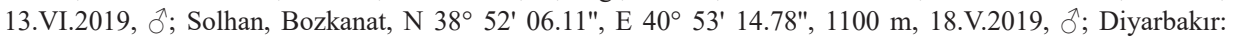

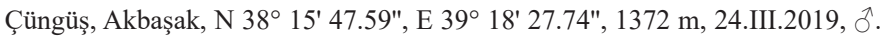

\section{Genus: Tachyancistrocerus Giordani Soika, 1957}

Tachyancistrocerus schmidti (Kokujev, 1912) (Fig. 4c)

Material examined: Bingöl: Genç, Doğanca, N 38 42' 49.25", E 40v 32' 17.06", $1135 \mathrm{~m}, 21$. VII.2017,, .

\section{Subfamily: Masarinae \\ Genus: Ceramius Latreille, 1810}

Ceramius palaestinensis (Giordani Soika, 1957) (Fig. 4d)

Material examined: Bingöl: İncesuyu, N 38 51' 41.86", E 40v 37' 27.74", 1065 m, 05.V.2018, क; Karlıova, Kızılçubuk, N 39² 23' 17.89", E 40 50' 47.87", 1874 m, 28.V.2017, ๆ; Solhan, Yenibaşak Bucağı, N $38^{\circ} 48^{\prime}$

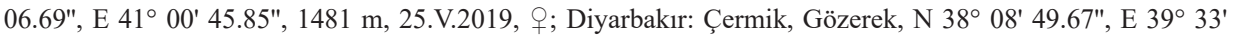

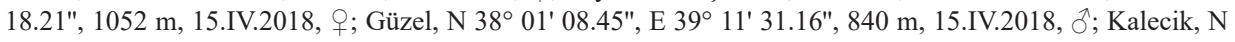
$38^{\circ} 06^{\prime} 46.52^{\prime \prime}$, E $40^{\circ} 29^{\prime} 32.35^{\prime \prime}, 906$ m, 13.IV.2018, o ; Çüngüs, Kayapınar, N 38 18' 28.44", E 39 11' 32.32",

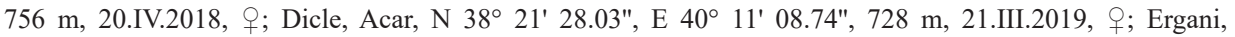
Çayırdere, N $38^{\circ} 11^{\prime} 50.80^{\prime \prime}$, E 39॰ 34' 19.42", 803 m, 21.IV.2018, ố; Dağlararası, N $38^{\circ} 13^{\prime} 08.55^{\prime \prime}$, E $39^{\circ} 35^{\prime}$ 55.84", 1029 m, 21.IV.2018, ; Salar, N 38 15' 41.31", E 39 41' 26.43", 899 m, 21.IV.2018, ơं; Hazro,

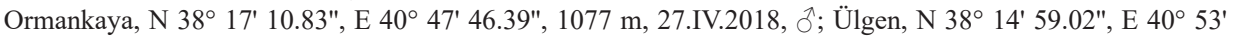
17.26", 989 m, 27.IV.2018, क; Kulp, Güllük, N 38 28' 35.13", E 40 54' 37.84", 869 m, 29.IV.2018, ค; Lice, Angül, N $38^{\circ} 23^{\prime} 13.08^{\prime \prime}$, E $40^{\circ} 32^{\prime} 56.04^{\prime \prime}, 877$ m, 25.IV.2018, o ; Silvan, Gündüz, N $38^{\circ} 16^{\prime} 29.97^{\prime \prime}$, E $39^{\circ} 55^{\prime}$ 10.39", $911 \mathrm{~m}, 29 . \mathrm{IV} .2018$, ô.

Subfamily: Polistinae

Genus: Polistes Latreille", 1802

Polistes atrimandibularis Zimmermann, 1930 (Fig. 4e)

Material examined: Bingöl: Kiğı, Duranlar, N 39 15' 46.46", E 40² 21' 01.32", 1207 m, 13.VI.2019, ㅇ.

Polistes biglumis alpium Blüthgen, 1957 (Fig. 4f)

Material examined: Muş: Bulanık, Gülova, N 3904' 00.80", E 42 19' 27.59", 1564 m, 02.VI.2017, q.

Polistes dominulus (Christ, 1791) (Fig. 4g)

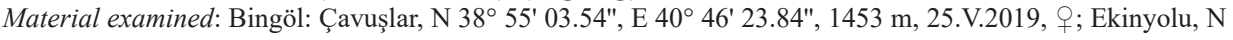



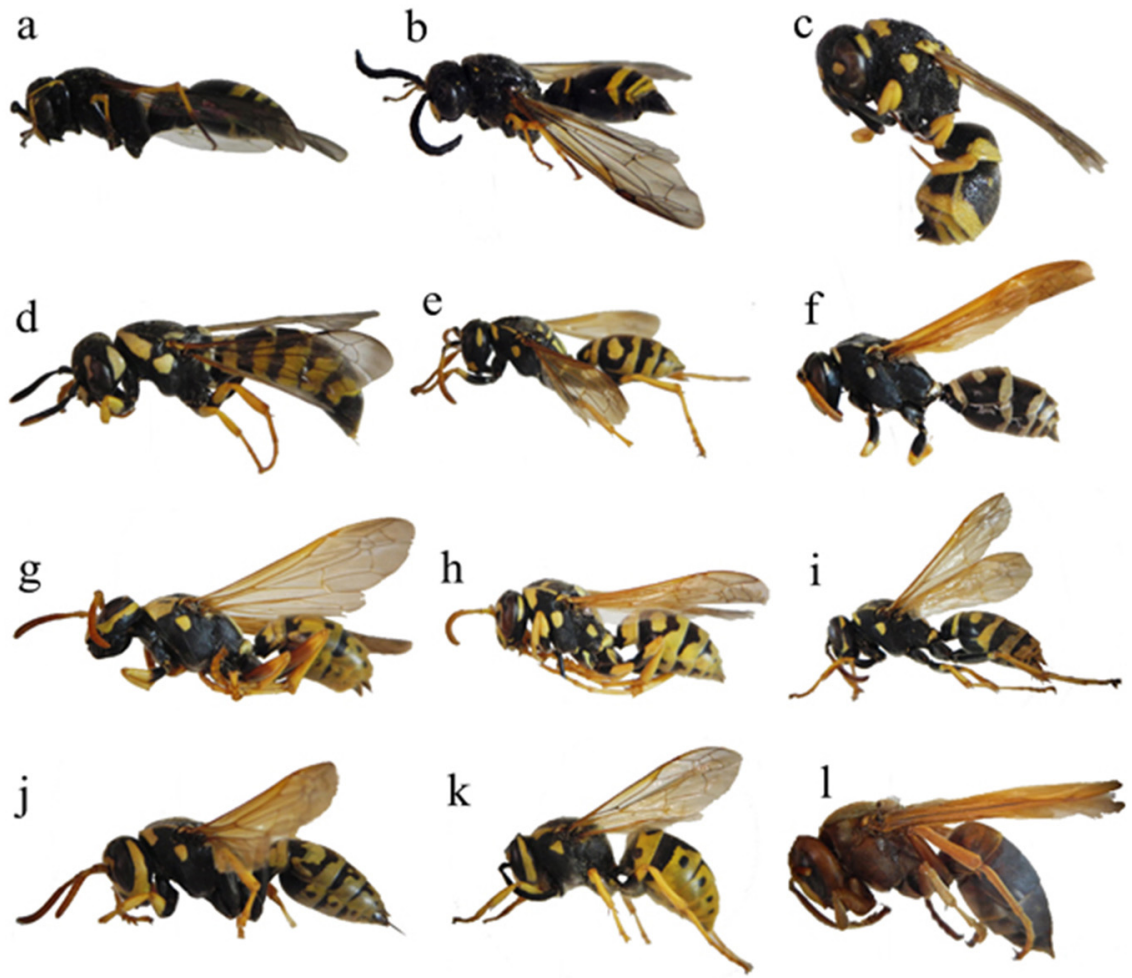

Fig. 4: a) Symmorphus gracilis (ঐ); b) S. murarius (ঐ); c) Tachyancistrocerus schmidti (†); d) Ceramius palaestinensis ( $($ ); e) Polistes atrimandibularis $(+)$; f) P. biglumis alpium ( $९)$; g) $P$. dominulus ( $($ ); h) P. gallicus ( $(+)$; i) P. nimpha $(+)$; j) P. sulcifer $(+)$; k) Vespula germanica $(\uparrow)$; l) V. orientalis ( + )

$38^{\circ} 53^{\prime} 53.54^{\prime \prime}$, E $40^{\circ} 34^{\prime} 31.81^{\prime \prime}, 1033$ m, 17.V.2018, o ; Haziran, N 38 49' 13.15", E 40 26' 57.10", 1672 m,

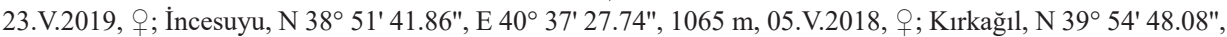

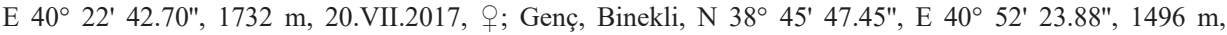

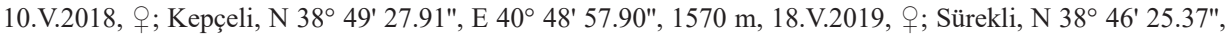
E $40^{\circ} 36^{\prime}$ 49.76", 1095 m, 18.V.2019, क; Yiğitbaşı, N 38 44' 48.62", E 40 45' 47.77", 1695 m, 21.VII.2017, o; Karlıova, Kaşıkçı, N 39 24' 12.94", E 41 00' 20.64", 1917 m, 01.VI.2019, o; Sarıkuşak, N 39 23' 27.29",

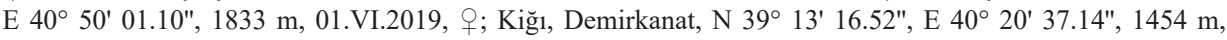

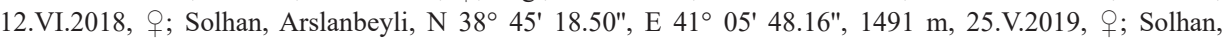

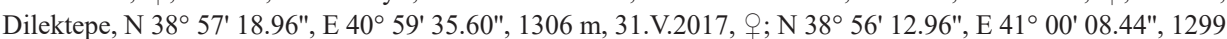
m, 24.V.2019, o ; Yayladere, Akçadamar, N 39 12' 11.02", E 40 04' 08.93", 1385 m, 13.VI.2019, o ; Kalkanlı,

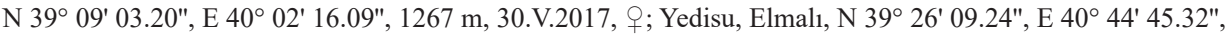

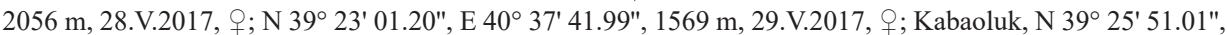

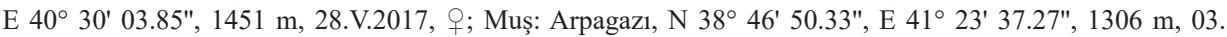
VI.2017, o; Bulanık, Akçaarmut, N 38 54' 14.17", E 41 58' 10.43", 1722 m, 02.VI.2017, o ; Kırkgöze, N $39^{\circ}$ 00' 35.69", E 42 22' 24.73", 1635 m, 02.VI.2017, क; Korkut, Pınarüstü, N $38^{\circ}$ 44' 37.44", E 41 45' 47.24", 1303 m, 02.VI.2017, o; Diyarbakır: Avcısuyu, N 38 03' 44.55", E 39 59' 22.13", 741 m, 23.III.2019, 六; Dicle, N $38^{\circ} 23^{\prime} 10.86^{\prime \prime}$, E $40^{\circ}$ 17' 29.72", 913 m, 28.III.2018, o+; Ergani, Yolköprü, N 38 15' 03.79", E 39

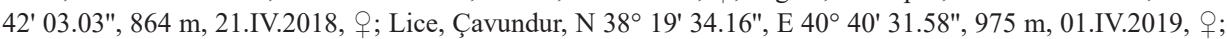


Kutlu, N $38^{\circ} 21^{\prime}$ 36.47", E 40 46' 45.42", 798 m, 12.IV.2019, +; Yalaza, N $38^{\circ}$ 20' 07.75", E 40 41' 03.75", 919 m, 01.IV.2019, o; Yolçat1, N 38² 23' 50.16", E 40 41' 02.65", 910 m, 20.V.2017, o; Silvan, Yeşilköy, N $38^{\circ} 06^{\prime} 50.15^{\prime \prime}, \mathrm{E} 41^{\circ} 08^{\prime}$ 51.27", 696 m, 15.V.2017, ‥

Polistes gallicus Linnaeus, 1761 (Fig. 4h)

Material examined: Bingöl: Altınışık, N 38 49' 41.39", E 40²7' 31.72", 1511 m, 23.V.2019, q; Çayağzı, N $38^{\circ} 48^{\prime}$ 21.94", E 40 33' 16.50", 1005 m, 24.V.2019, , ; Elmalı, N 39 02' 11.07", E 40 44' 45.03", 1280 m,

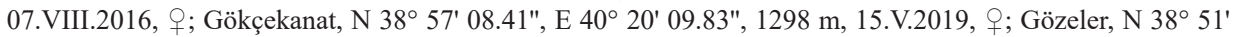
17.14", E 40 40' 36.42", 1153 m, 25.V.2019, ,; Güveçli, N $38^{\circ}$ 51' 17.54", E 40 31' 57.43", 1050 m,

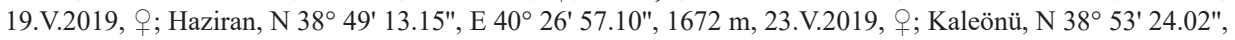

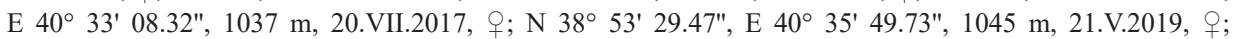

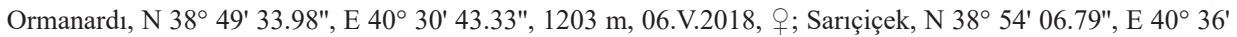
37.86", 1059 m, 17.V.2018, , ; Seitgölü, N 3904' 51.31", E 40² 20' 07.71", 1613 m, 27.V.2017, q; Sudüğünü, N 39॰ 03' 32.78", E 40²4' 11.34", 1582 m, 05.VIII.2016, o; Üçyaka, N $38^{\circ} 50^{\prime}$ 57.05", E 40 27' 09.72", 1701 m, 23.V.2019, +; Adakl1, N 39 12' 59.81", E 40²8' 09.21", 1360 m, 12.VI.2019, +; Arica, N 39 14' 00.32", E 40 31' 38.49", 1324 m, 12.VI.2019, o; Dayankaya, N 39 11' 08.33", E 40 34' 54.57", 1675 m, 21.V.2017,

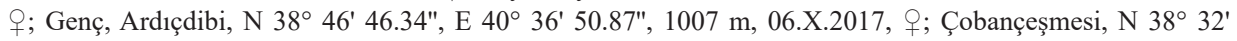
35.75", E 40 16' 48.42", 868 m, 17.V.2019, +; Doğanca, N 38 42' 51.19", E 40 32' 44.66", 1164 m,

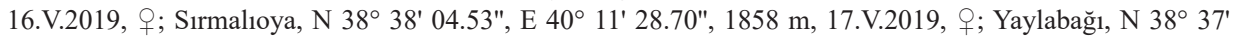
45.24", E 40 30' 45.83", 1247 m, 26.V.2017, +; Yoldaşan, N 38 43' 88.00", E 40 29' 15.49", 997 m, 05.V.2017, o; Karliova, N 39 12' 33.66", E 40 57' 24.32", 1734 m, 03.VI.2018, o; Suçat1, N 39²3' 09.50", E 40 54' 35.15", 1805 m, 28.V.2017, o.; Taşlıçay, N 39 16' 28.62", E 40 58' 56.74", 1852 m, 28.VIII.2016, o; Kiğı, Çanakal, N $39^{\circ} 09^{\prime}$ 16.98", E 40 18' 43.73", 1585 m, 13.VI.2019, + ; Sabırtaş1, N 39 15' 56.52", E

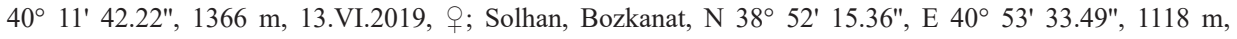
26.V.2019, ㅇ; N $38^{\circ}$ 52' 06.11", E 40 53' 14.78", 1100 m, 18.V.2019, ㅇ; N 39 15' 32.11", E 40 19' 04.67", 1358 m, 13.VI.2019, o+; Çevrimpınar, N 38 51' 03.96", E 40 58' 48.67", 1183 m, 26.V.2019, + ; Yayladere,

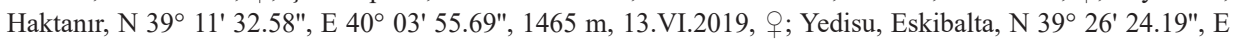
$40^{\circ} 28^{\prime}$ 16.59", 1415 m, 06.VI.2019, o; Kabaoluk, N 39v 25' 55.09", E 40²9' 59.21", 1412 m, 06.VII.2018, ૧; Şenköy, N 39v 25' 17.82", E 40 31' 48.37", 1493 m, 06.VII.2018, ㅇ; Diyarbakır: Bismil, Göksu, N $37^{\circ} 50^{\prime}$ 00.00", E 40³1' 40.57", 550 m, 19.III.2019, o; Köseli, N 37 50' 51.99", E 40 36' 49.64", 558 m, 30.III.2018,

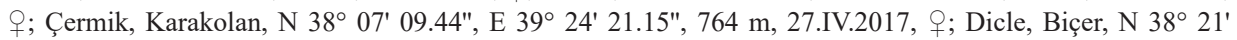
59.34", E 40 01' 33.98", 957 m, 20.IV.2018, , ; Hazro, Uzunargit, N 38 14' 07.05", E 40 46' 58.96", 942 m, 04.IV.2019, +; Kocaköy, Yazıköy, N $38^{\circ}$ 18' 55.71", E 40 31' 21.30", 901 m, 01.IV.2019, , ; Lice, Oyuklu, N

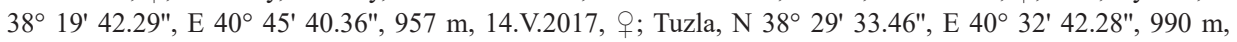
28.V.2016, +; Silvan, Babakaya, N $38^{\circ}$ 14' 40.39", E 4106' 48.67", 787 m, 05.IV.2019, q; Muş: Hasköy, N

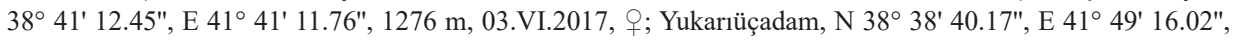
1276 m, 03.VI.2017, o; Korkut, Oğulbalı, N $38^{\circ}$ 43' 25.44", E 41 49' 43.09", 1297 m, 02.VI.2017, q.

\section{Polistes nimpha (Christ, 1791) (Fig. 4i)}

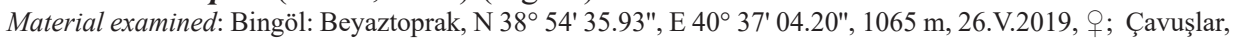
N $38^{\circ}$ 55' 03.54", E 40 46' 23.84", 1453 m, 25.V.2019, ㅇ; Haziran, N 38 49' 13.15", E 40²6' 57.10", 1672 m, 23.V.2019, ; Kaleönü, N 38 53' 28.91", E 40 33' 25.74", 1025 m, 21.V.2019, ,; Köklü, N $38^{\circ} 56^{\prime} 00.43^{\prime \prime}$, E $40^{\circ} 38^{\prime}$ 48.68", 1092 m, 19.V.2018, q; Kardeşler, N $38^{\circ}$ 54' 53.33", E 40 37' 24.74", 1068 m, 17.V.2018, , ; Küçükterkören, N $38^{\circ} 49^{\prime} 49.46^{\prime \prime}$, E 40 34' 28.59", 1009 m, 16.V.2019, o; N 38 50' 41.90", E 40³0' 17.75", 1156 m, 06.V.2018, +; N $38^{\circ}$ 51' 01.40", E 40 30' 02.62", 1182 m, 19.V.2019, , ; Nacakl1, N 39 10' 05.38",

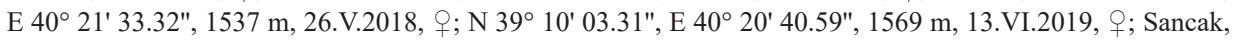

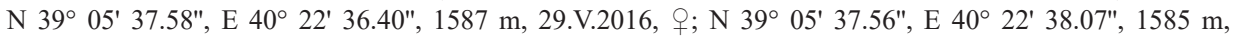
26.V.2018, +; Sarıçiçek, N $38^{\circ}$ 53' 02.44", E 40 35' 02.83", 1057 m, 19.V.2019, q; Sudüğünü, N 390 03' 32.78", E 40²4' 11.34", 1582 m, 05.VIII.2016, ơ; Yamaç Bucağ1, N 38 46' 12.67", E 40²5' 59.75", 1364 m, 23.V.2019, o; Yeşilköy, N $38^{\circ}$ 50' 20.35", E 40²9' 44.38", 1280 m, 31.V.2020, o; Yukarıpınar, N $38^{\circ} 50^{\prime}$ 57.05", E 40²7' 09.72", 1701 m, 23.V.2019, .;; Adakl1, Gökçeli, N 39 11' 50.31", E 40²5' 15.91", 1626 m, 11.VI.2018, +; Genç, Dikpınar, N 38 43' 24.50", E 40 18' 35.18", 975 m, 17.V.2019, q; Şehitköy, N 38 42' 04.23", E 40 30' 41.61", 1147 m, 14.V.2018, .; Karlıva, Kaynak, N 39 14' 09.49", E 40 54' 41.31", 1897 m, 31.V.2019, o; Kıraçtepe, N 39 12' 33.66", E 40 57' 24.32", 1734 m, 03.VI.2018, of; Çilligöl, N 39 10' 13.31", E 40 55' 33.91", 1854 m, 31.V.2019, ㅇ; Kiğı1, Sabırtaşı, N 39 15' 56.52", E 40 11' $42.22^{\prime \prime}, 1366$ m, 


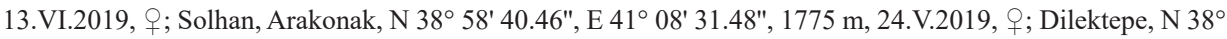
56' 56.65", E 40 59' 15.12", 1278 m, 24.V.2019, ㅇ; Elbaşı, N $38^{\circ} 59^{\prime}$ 08.01", E 41 03' 15.73", 1583 m, 24.V.2019, o; Kale, N $38^{\circ} 47^{\prime}$ 17.38", E 4102' 28.54", 1312 m, 25.V.2019, o; Mutluca, N 3901' 10.17", E $40^{\circ}$ 52' 01.39", 1631 m, 26.V.2019, ㅇ; Yenidal, N 38 54' 29.31", E 40 55' 54.28", 1114 m, 18.V.2019, ㅇ;

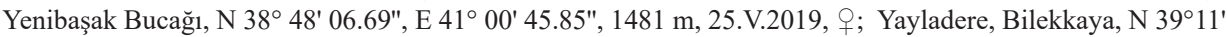
23.75", E 40 11' 08.71", 1453 m, 30.V.2017, o; Doyucak, N 39॰ 09' 46.49", E 40 64' 27.75", 1462 m, 13. VI.2019, o; Kalkanlı, N 390 09' 03.64", E 40 01' 50.85", 1401 m, 30.V.2017, o ; Zeyneli, N 39 12' 18.24", E 40॰ 09' 49.04", 1363 m, 13.VI.2019, o; Yedisu, Elmalı, N 39²2' 04.62", E 40 39' 12.28", 1885 m, 06.

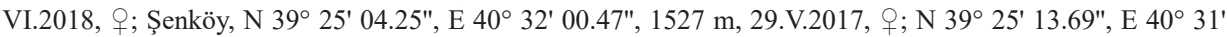
52.37", 1496 m, 06.VI.2018, o ; Diyarbakır: Köprübaş1, N 37 59' 64.39", E 40²2' 59.69", 602 m, 21.III.2019,

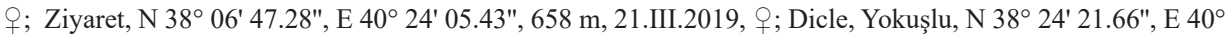
00' 56.08", 798 m, 28.III.2019, , ; Eğil, N 38 14' 54.73", E 40 06' 08.25", 801 m, 28.III.2019, , \&; Ergani, Boğazköy, N 38 15' 45.62", E 39 41' 21.63", 890 m, 24.III.2019, , ; Hani, Yukarıturalı, N $38^{\circ} 28^{\prime}$ 22.43", E 40²7' 13.81", 988 m, 27.III.2019, of; Hazro, Ormankaya, N 38 18' 13.20", E 40 44' 29.38", 1099 m, 19.

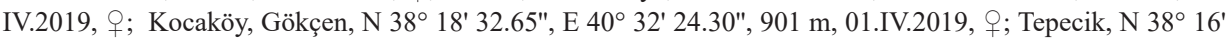

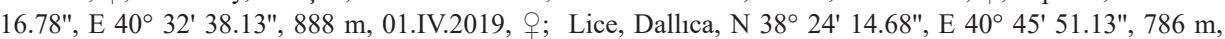
12.IV.2019, o; Daralan, N $38^{\circ} 27^{\prime}$ 46.65", E 40³2' 06.82", 1400 m, 18.IV.2019, o; Kabakaya, N $38^{\circ} 20^{\prime}$ 12.94", E 40 44' 06.70", 839 m, 11.IV.2019, + ; Oyuklu, N $38^{\circ}$ 19' 55.42", E 40 45' 05.76", 925 m, 11.IV.2019, +; Savat Bucağı, N 38²1' 03.07", E 40³9' 10.00", 923 m, 19.IV.2019, q.

Polistes sulcifer Zimmermann, 1930 (Fig. 4j)

Material examined: Bingöl: Genç, Ericek, N 38 34' 05.02", E 40 15' 15.02", 1067 m, 17.V.2019, , ; Solhan, Arakonak, N $38^{\circ} 58^{\prime}$ 40.46", E $41^{\circ} 08^{\prime} 31.48^{\prime \prime}, 1775$ m, 24.V.2019, o.

\section{Subfamily: Vespinae Latreille, 1802 Genus: Vespula Thomson», 1869}

\section{Vespula germanica (Fabricius, 1793) (Fig. 4k)}

Material examined: Bingöl: Çayağzı, N $38^{\circ}$ 47' 44.47", E 40³3' 15.35", 998 m, 20.V.2018, q; Çeltiksuyu, N $38^{\circ}$ 51' 06.77", E 40 33' 56.61", 1013 m, 24.V.2019, o; Ekinyolu, N 38 54' 13.80", E 40 35' 10.08", 1040

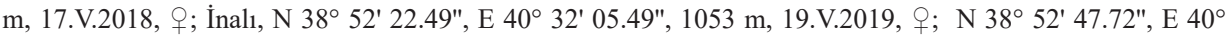
32' 33.82", 1030 m, 20.V.2019, + ; Sarıçiçek, N 38 53' 43.75", E 40 35' 56.25", 1045 m, 16.V.2019, ㅇ;

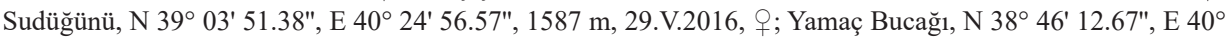

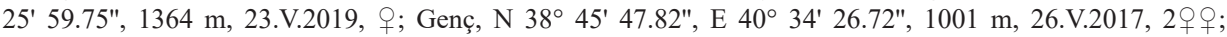
Çanakal, N $38^{\circ} 45^{\prime}$ 55.39", E 40 43' 58.57", 1530 m, 26.V.2017, q. Kartal, N 390 01' 05.56", E 40²3' 28.63", 1675 m, 26.V.2018, o; Komgeçit, N $38^{\circ}$ 47' 45.84", E 40³7' 57.35", 1003 m, 09.V.2018, o.; Sülünkaş, N 38º 49' 38.68", E 40 56' 57.07", 1647 m, 25.V.2019, o; Yayla Bucağ1, N 38 38' 20.25", E 40 31' 57.34", 1369 m, 16.V.2019, +; Karlıova, Hacilar, N 39 05' 01.63", E 40 49' 21.67", 1417 m, 31.V.2019, +; Kaynarpinar, N 39 23' 02.82", E 40 45' 42.74", 1767 m, 01.VI.2019, , ; Yeniköy, N 39 11' 50.05", E 40 56' 07.35", 1748 m, 03.VI.2018, ๆ; Kiğı, Yeşilyurt, N 39 16' 58.79", E 40²1' 24.37", 1323 m, 12.VI.2018, o ; Solhan, Arakonak, N $38^{\circ}$ 56' 49.12", E 41 07' 40.34", 1660 m, 24.V.2019, , ; Yayladere, N 39 13' 11.16", E 4004' 10.77", 1553 m, 30.V.2017, q; Yaylabağ, N 39 11' 11.13", E 40 06' 17.29", 1379 m, 13.VI.2018, , Yedisu, Gelinpertek, N $39^{\circ} 27^{\prime}$ 24.30", E 40 24' 24.93", 1410 m, 28.V.2017, o; Diyarbakır: Çüngüş, Güneydere, N $38^{\circ} 13^{\prime}$ 31.61", E $39^{\circ} 18^{\prime}$ 57.67", 1099 m, 28.IV.2017, o; Dicle, Değirmeneli, N 38² 24' 37.51", E 40v 11' 42.67", 1255 m, 27.III.2019, q; Hani, Kırım, N $38^{\circ}$ 23' 44.75", E 40v 26' 24.13", 863 m, 27.III.2019, O; Uzunlar, N 38v 24' 34.34", E 40²7' 03.41", 923 m, 27.III.2019, , ; Hazro, Kavakl1, N 38 57' 36.43", E 41 00' 48.83", 1333 m, 24.V.2019, ,; Kocaköy, N 38 16' 03.92", E 40 34' 20.94", 828 m, 28.V.2016, q; Tepecik, N $38^{\circ}$ 16' 03.92", E 40 34' 20.94", 828 m, 28.V.2016, + ; Muş: Hasköy, N 38 41' 12.45", E 41 41' 11.76",

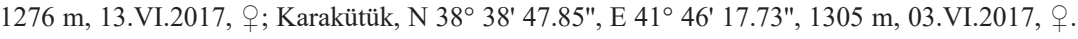

Vespula orientalis (Linnaeus, 1771) (Fig. 41)

Material examined: Bingöl: Genç, Çanakçı, N 38 47' 07.85", E 40 43' 50.07", 1312 m, 18.V.2019, ㅇ; Dedebağı, N $38^{\circ} 40^{\prime} 37.61 "$ ", E 40 19' 33.71", 1234 m, 16.V.2019, , ; Dikköy, N 38 48' 42.96", E 40³9' 50.00", 1012 m, 09.V.2018, q; Diyarbakır: Dicle, N 37 52' 50.22", E 40 14' 33.39", 625 m, 30.IV.2016, q. 


\section{Acknowledgements}

We would like to thank Bingol University and Atatürk University for their contributions to laboratory studies.

\section{References}

Carpenter, G. 1911: "Hymenoptera" . In Chisholm, Hugh (ed.). Encyclopaedia Britannica. - Cambridge University Press 11(14): 177.

Kaplan, E., Mart, A., Haris, A. \& Yildirim, E. 2018: Contribution to the knowledge of the Megalodontesidae, Argidae, Cimbicidae, Cephidae and Tenthredinidae (Symphyta: Hymenoptera) fauna from Bingöl and Diyarbakır provinces of Turkey. - Natura Somogyiensis Kaposvár 32: 109-120. https://doi.org/10.24394/ NatSom.2018.32.109

Lima, A. \& Dias, P. 2018: The New World species of Leucospis Fabricius, 1775 (Hymenoptera, Chalcidoidea, Leucospidae): an update of Bouček's revision with description of two new species from Brazil. - Zootaxa 4441. https://doi.org/10.11646/zootaxa.4441.1.1

Ljubomirov, T. \& Yildirim, E. 2008: Annotated catalogue of the Ampulicidae, Sphecidae ve Crabronidae (Insecta: Hymenoptera) of Turkey. - Pensoft Series 71: 1312- 0174.

Noyes, J. S. 2018: Universal Chalcidoidea Database. World wide web electronic publication. http:// www.nhm. ac.uk/chalcidoids.

YILDIRIM, E. 2012: The distribution and biogeography of Vespidae (Hymenoptera: Aculeata) in Turkey. Turkish Journal of Entomology 36: 23-42.

YILDIRIM, E. 2016: Overview of the distribution and biogeography of Sapygidae (Hymenoptera: Aculeata) in Turkey. - Entomofauna 37: 565-572.

Yildirim, E. \& Gusenleitner, J. 2012: Contribution to the knowledge of the Vespidae (Hymenoptera, Aculeata) of Turkey, with a checklist of the Turkish species. - Turkish Journal of Zoology 36 (3): 361-374. https://doi.org/10.3906/zoo-1012-62

Yildirim, E. \& Gusenleitner, J. 2015: The present situation of the Vespidae-fauna (Hymenoptera, Aculeata) of Turkey. - Linzer biologische Beiträge 47: 991-1002. 
